\title{
An infrared study of pure and ion irradiated frozen formamide
}

\author{
J. R. Brucato ${ }^{1}$, G. A. Baratta ${ }^{2}$, and G. Strazzulla ${ }^{2}$ \\ 1 INAF - Osservatorio Astronomico di Capodimonte, via Moiariello 16, 80131 Napoli, Italy \\ e-mail: brucato@na.astro.it \\ 2 INAF - Osservatorio Astrofisico di Catania, via Santa Sofia 78, 95123 Catania, Italy
}

Received 27 February 2006 / Accepted 10 April 2006

ABSTRACT

\begin{abstract}
Context. The chemical evolution of formamide $\left(\mathrm{HCONH}_{2}\right)$, a molecule of astrobiological interest that has been tentatively identified in interstellar ices and in cometary coma, has been studied in laboratory under simulated astrophysical conditions such as ion irradiation at low temperature.

Aims. To evaluate the abundances of formamide observed in space or in laboratory, the integrated absorbances for all the principal IR features of frozen amorphous pure formamide deposited at $20 \mathrm{~K}$ were measured. Further evidence that energetic processing of ices occurring in space is extremely relevant both to astrochemistry and to astrobiology has been found, showing that new molecular species are synthesized by ion irradiation at a low temperature.

Methods. Pure formamide were deposited at $20 \mathrm{~K}$ and IR transmission spectra measured for different ice thicknesses. The ice thickness was derived by looking at the interference pattern (intensity versus time) of a $\mathrm{He}-\mathrm{Ne}$ laser beam reflected at an angle of 45 deg by the vacuum-film and film-substrate interfaces. Samples of formamide ice were irradiated with $200 \mathrm{keV} \mathrm{H}^{+}$ions and IR spectra recorded at different ion fluences.

Results. New molecules were synthesized among which are $\mathrm{CO}, \mathrm{CO}_{2}, \mathrm{~N}_{2} \mathrm{O}$, isocyanic acid ( $\mathrm{HNCO}$ ), and ammonium cyanate $\left(\mathrm{NH}_{4}{ }^{+} \mathrm{OCN}^{-}\right)$. Some of these species remain stable after warming up to room temperature.
\end{abstract}

Key words. astrochemistry - astrobiology - methods: laboratory - techniques: spectroscopic - ISM: molecules - comets: general

\section{Introduction}

Dust particles are mostly formed in circumstellar regions of evolved stars and ejected in the interstellar medium. In dense molecular clouds, most of the gas-phase species are rapidly condensed on refractory cores, forming icy mantles. The frozen mantles are rich in water, the dominant ice in the Universe, which was identified as a component of dust grains (Willner et al. 1982). Space-based infrared observations performed by observatories such as ISO or Spitzer show that new icy species are continuously detected.

Formamide $\left(\mathrm{HCONH}_{2}\right)$ was observed in the interstellar medium (for a review of molecules observed in interstellar and circumstellar media, see Millar 2004), in the long period comet C/1995 O1 Hale-Bopp (Bockeleé-Morvan 2000), and tentatively in young stellar objects W33A (Schutte et al. 1999) and NGC 7538 IRS9 (Raunier et al. 2004). Formamide is very interesting for its active role in prebiotic chemistry. The chemical reactions of simple compounds containing $\mathrm{H}, \mathrm{C}, \mathrm{N}$, and $\mathrm{O}$, such as formamide, are considered a plausible pathway for synthesis on the Earth of biomolecules under prebiotic conditions (Oparin 1938; Miller 1953; Eschenmoser \& Loewenthal 1992; Chyba \& McDonald 1995; Saladino et al. 2004). Among the different theories of prebiotic chemistry active in the early Earth (Miller-Urey and Fischer-Tropsh synthesis), the extraterrestrial synthesis of organic compounds and delivery on the planetary surface is an interesting one. A considerable amount of extraterrestrial material was and is continuously delivered on the Earth. It was estimated that between $10^{7}$ and $10^{9} \mathrm{~kg} \mathrm{yr}^{-1}$ of carbon contained in organic compounds arrived here in the first billion years as interplanetary dust particles (IDPs) (Chyba \& Sagan 1992). Moreover, different strategies for synthesizing organic molecules occurring in space consider simple nitrogen-bearing compounds - such as hydrogen cyanide $(\mathrm{HCN})$, isocyanic acid ( $\mathrm{HNCO}$ ), formamide, ammonium cyanide $\left(\mathrm{NH}_{4} \mathrm{CN}\right)$, or mixtures of $\mathrm{H}_{2} \mathrm{O}, \mathrm{CO}_{2}, \mathrm{CO}, \mathrm{NH}_{3}, \mathrm{CH}_{4}, \mathrm{CH}_{2} \mathrm{O}, \mathrm{CH}_{3} \mathrm{OH}$, etc. - as potential astrobiological precursors (Grim \& Greenberg 1987; Demyk et al. 1998; Moore \& Hudson 2003; Hudson et al. 2001; Palumbo et al. 2000, 2004). In this work we focused on pure formamide molecule under simulated astrophysical conditions.

The $\mathrm{HCONH}_{2}$ molecule is formed at room temperature by the hydrolysis of $\mathrm{HCN}$, and it is the most abundant product of the pyrolysis of HCN-polymer. It is a reactive compound both at the carboxyl moiety and at the amino group. The role of formamide as prebiotic precursor of the synthesis of nucleic acid bases has been shown under a variety of conditions. In particular the presence of different inorganic compounds, such as oxides, minerals or cosmic dust analogues, is able to catalyze the formamide condensation that originate, among many compounds, purine and pyrimidine bases, namely, the products of the first primordial steps that might have leaded to the appearance of life as we know it (Yamada et al. 1972, 1975; Saladino et al. 2003, 2004, 2005).

Energetic processing of ices occurring in space is extremely relevant both to astrochemistry and to astrobiology. Processing by UV photons of interstellar/cometary ice analogue mixtures of $\mathrm{H}_{2} \mathrm{O}, \mathrm{CH}_{3} \mathrm{OH}, \mathrm{CO}$, and $\mathrm{NH}_{3}$ has shown that during warming up to $200 \mathrm{~K}$ formamide, acetamide, ethanol, and nitriles compounds are formed (Bernstein et al. 1995). Moreover, it was suggested that formamide is one of the products of the ion 
irradiation and phoyolysis of $\mathrm{H}_{2} \mathrm{O}+\mathrm{HCN}$ (Gerakines et al. 2004) or of $\mathrm{NH}_{3}+\mathrm{CO}$ (Demyk et al. 1998), even at a low temperature $(18 \mathrm{~K})$. Unfortunately, a quantitative measurement of the amount synthesized has not been made due to lacking IR band absorbances. Experimental investigations of chemical evolution of pure formamide in simulated interstellar/cometary conditions are therefore deemed necessary.

In this paper we present an IR transmittance spectroscopy study in the $4000-1200 \mathrm{~cm}^{-1}$ range of pure formamide deposited at a low temperature $(20 \mathrm{~K})$. We measured the absorbances of the major IR bands and irradiated samples of condensed formamide with $200 \mathrm{keV} \mathrm{H}^{+}$ions at different fluences. New molecules were synthesized and identified in the spectra. The chemical evolution of the irradiated samples were then monitored at increasing temperatures.

\section{Experimental procedures}

Gaseous samples of formamide were prepared in a pre-chamber $\left(P<10^{-6}\right.$ mbar $)$ and admitted by a gas inlet through a needle valve into the scattering chamber, where they accreted onto a cold $(20 \mathrm{~K})$ silicon substrate; the thickness of the deposited films were then measured (see below for details). The infrared transmittance spectra were obtained in the high-vacuum scattering chamber $\left(P<10^{-7}\right.$ mbar $)$ interfaced with a FTIR spectrophotometer (Bruker Equinox 55) through IR-transparent windows. The silicon substrate was in thermal contact with a closed-cycle helium cryostat whose temperature can be varied between 10 and $300 \mathrm{~K}$. The vacuum chamber is interfaced with an ion implanter (200 kV; Danfysik 1080-200) that generates ions with energies up to $200 \mathrm{keV}$ (400 keV for double ionization). The ion beam produces a spot that is larger than the area probed by the infrared beam (for more details on the experimental set up see Strazzulla et al. 2001). In this work we used $200 \mathrm{keV}$ $\mathrm{H}^{+}$ions. In order to avoid macroscopic heating of the target, the current density was maintained in the range of $100 \mathrm{nA} \mathrm{cm}^{-2}$ to a few $\mu \mathrm{A} \mathrm{cm}^{-2}$. The ion fluence in ions $\mathrm{cm}^{-2}$ was measured by a charge integrator from the ion current monitored during irradiation. The substrate plane is placed at an angle of 45 degrees with respect to the IR beam and the ion beam so that spectra can be taken in situ, even during irradiation, without tilting the sample. Spectra were taken at selected temperatures in the range of $20-300 \mathrm{~K}$. All the spectra shown below were taken with a resolution of $1 \mathrm{~cm}^{-1}$ using a DTGS detector.

The $200 \mathrm{keV} \mathrm{H}^{+}$ions penetrate about $2 \mu \mathrm{m}$ in formamide calculated using the TRIM program (Transport of Ions in Matter; e.g., Ziegler 1977; Ziegler et al. 1996). The maximum thickness of deposited layers was about $0.49 \mu \mathrm{m}$ (see below) i.e. thinner than the penetration depth of incoming ions. With the same software, it is also possible to calculate the stopping power (i.e. the amount of energy deposited per unit path length) of a given ion in a given target. For $200 \mathrm{keV} \mathrm{H} \mathrm{H}^{+}$in formamide we obtained about $50 \times 10^{-15} \mathrm{eV} \mathrm{cm}^{2} /$ molecula. By multiplying this number times the number of bombarding ions per square centimetre we obtained the amount of energy released to the sample (dose) in $\mathrm{eV}$ per molecula. Here we express the dose in eV/16amu, because it is a convenient way to characterize chemical changes and to compare with other experiments with different samples.

\section{Results}

Figure 1 shows the two spectra of $\left(\mathrm{HCONH}_{2}\right)$ before and after ion irradiation $\left(12 \mathrm{eV} / 16 \mathrm{amu}\right.$ deposited by $200 \mathrm{keV} \mathrm{H}^{+}$ions $)$

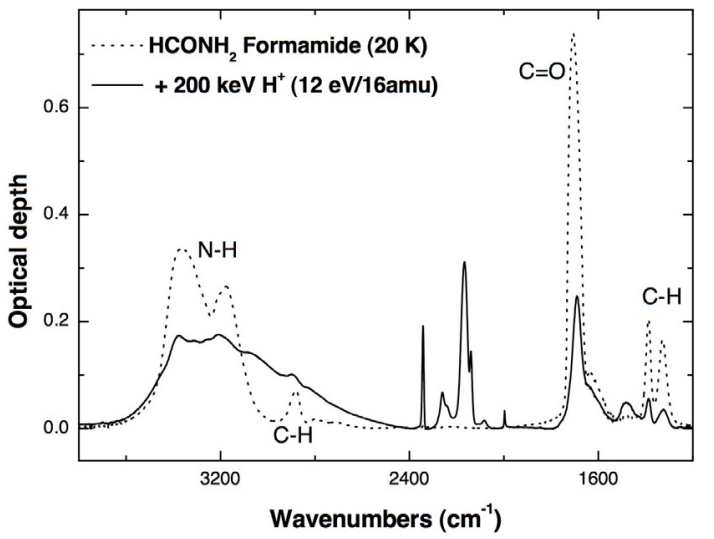

Fig. 1. IR transmittance spectrum in optical depth scale of formamide as deposited at $20 \mathrm{~K}$ on a silicon substrate (dot line). The band-peak positions and assignment are given in Table 1 . The spectrum obtained after irradiation at a dose of $12 \mathrm{eV} / 16 \mathrm{amu}$ with $200 \mathrm{keV} \mathrm{H}^{+}$ions is also shown (full line).

Table 1. Peak positions, vibration assignments, and integrated absorbances of frozen $(20 \mathrm{~K})$ amorphous formamide.

\begin{tabular}{ccll}
\hline \hline $\begin{array}{c}\text { Peak position } \\
\mathrm{cm}^{-1}\end{array}$ & $\mu \mathrm{m}$ & Assignment & $\begin{array}{l}\text { Integrated absorbance } \\
\left(\times 10^{-17} \mathrm{~cm} \mathrm{~mol}^{-1}\right)\end{array}$ \\
\hline 3368 & 2.97 & $v_{1}$ asym. $\mathrm{NH}_{2}$ stretch & $13.49\left(v_{1}+v_{2}\right)$ \\
3181 & 3.14 & $v_{2}$ sym. $\mathrm{NH}_{2}$ stretch & \\
2881 & 3.47 & $v_{3} \mathrm{CH}$ stretch & 0.47 \\
1708 & 5.85 & $v_{4} \mathrm{CO}$ stretch & $6.54\left(v_{4}+v_{5}\right)$ \\
1631 & 6.13 & $v_{5}$ in plane $\mathrm{NH}_{2}$ scissoring & \\
1388 & 7.20 & $v_{6}$ in plane $\mathrm{CH}$ scissoring & 0.68 \\
1328 & 7.53 & $v_{7} \mathrm{CN}$ stretch & 0.85 \\
\hline
\end{tabular}

at $20 \mathrm{~K}$ in the $3800-1200 \mathrm{~cm}^{-1}$ range. The peak positions of fundamental vibrational modes of formamide and their assignments are reported in Table 1 . Such assignments were made on the basis of work on gaseous or liquid samples (McNaughton et al. 1999; Rubalcava 1956). The $\left(\mathrm{HCONH}_{2}\right)$ spectrum is characterized by a couple of intense and broad bands at 3368 and $3181 \mathrm{~cm}^{-1}$, which correspond to asymmetric and symmetric $\mathrm{NH}_{2}$ stretching modes, respectively. The strongest band is peaked at $1708 \mathrm{~cm}^{-1}$ and related to the CO-stretching mode of the carbonyl group.

\subsection{Absorbances}

The experimental set-up allows the thickness to be monitored during accretion by looking at the interference pattern (intensity versus time) given by a He-Ne laser beam reflected at an angle of $45 \mathrm{deg}$ by the vacuum-film and film-substrate interfaces. After the reflection from the substrate, the laser beam follows the same path of the infrared beam coming from the IR source and can be detected by using an external detector placed in the source compartment of the IR spectrometer.

In general the interference curve versus thickness given by the laser beam reflected by the film+substrate assembly is an oscillating function. For absorbing materials, the laser light transmitted into the film and reflected back by the interface filmsubstrate is attenuated into the film; thus the amplitude of the oscillation in the interference curve exponentially decays with the thickness, and the reflectance approaches its bulk value at a large thickness. In molecular ices at visible wavelengths, the absorption is so low that it can be neglected for a thickness of a 
few microns, so that the interference curve can be considered a periodic function whose period (distance between two maxima or minima) is given by the relation:

$$
\Delta d=\frac{\lambda_{0}}{2 n_{\mathrm{f}} \sqrt{1-\sin ^{2} \theta_{\mathrm{i}} / n_{\mathrm{f}}^{2}}}
$$

where $n_{\mathrm{f}}$ is the refractive index of the ice at the laser wavelength $\lambda_{0}$, and $\theta_{\mathrm{i}}$ is the incidence angle measured from the normal. The previous relation can be used to measure the thickness if $n_{\mathrm{f}}$ is known. The amplitude of the interference curve depends on the refractive index of the ice, the refractive index of the substrate, the incidence angle, and on the polarization of the laser. Hence $n_{\mathrm{f}}$ can be derived from the amplitude of the interference curve (intensity ratio between maxima and minima); details of this method can be found in Westley et al. (1998) and Baratta \& Palumbo (1998).

The absolute accuracy of the thickness measured in this way is about $5 \%$ and is limited mainly by the uncertainties in the knowledge of the refractive index of the substrate at low temperature (silicon in our case) and by the error in measuring the incidence angle of the laser. By following this method we found a refractive index $n_{\mathrm{f}}=1.361$ for formamide ice at the laser wavelength of $0.543 \mu \mathrm{m}$. For an incidence angle of $45 \mathrm{deg}$, this yields a film thickness of $0.234 \mu \mathrm{m}$ between interference maxima. Once the thickness is measured, the integrated bands absorbance, $A\left(\mathrm{~cm} \mathrm{~mol}^{-1}\right)$, can be computed from the infrared spectrum if the density $\rho$ of the film is known through the formula:

$A=\frac{\int \tau(v) \mathrm{d} v}{N}$

where $\tau(v)$ is the optical depth and $N$ the molecular column density in units of molecules $\mathrm{cm}^{-2}$.

In this work we derived the density of formamide ice by using the Lorentz-Lorenz relation:

$L \rho=\frac{n_{\mathrm{f}}^{2}-1}{n_{\mathrm{f}}^{2}+2}$.

For a given material, the quantity $L$ (Lorentz-Lorenz coefficent) is nearly constant for a given wavelength regardless of the material phase and temperature (Wood \& Roux 1982). Formamide at $25{ }^{\circ} \mathrm{C}$ (liquid) has a density $\rho^{\text {liq }}=1.129 \mathrm{~g} \mathrm{~cm}^{-3}$ and a refractive index of $n_{\mathrm{f}}^{\text {liq }}=1.446$ for the sodium line $(0.589 \mu \mathrm{m})$ (Cases et al. 2001). The corresponding Lorentz-Lorenz coefficient is $L=0.2362 \mathrm{~cm}^{3} \mathrm{~g}^{-1}$. By substituting in Eq. (3), the $L$ coefficient derived for the liquid phase and the refractive index measured by interference, we obtain a density $\rho^{\text {ice }}=0.937 \mathrm{~g} \mathrm{~cm}^{-3}$ for formamide ice. The corresponding integrated band absorbances are given in Table 1, together with band peak positions and assignments. The absorbances were derived by considering two different thickness increased by a factor (from the Snell's low) of $1 / \cos \theta_{\mathrm{r}}=1 / \sqrt{1-\sin ^{2} \theta_{\mathrm{i}} / n_{\mathrm{f}}^{2}}$, where $\theta_{\mathrm{r}}$ is the refractive angle. The correction takes into account, in a approximate way, the increased path length of the IR beam at an oblique incidence of $\theta_{\mathrm{i}}=45 \mathrm{deg}$. The corrective factor was derived by assuming a constant value of the refractive index with the wavelength. This approximation neglects the variation of the refractive index in the infrared region due to the vibrations and the contribution of the electronic transitions to the dispersion from the visible $(0.543 \mu \mathrm{m})$ to the infrared. We estimate a corresponding uncertainty of $\simeq 10 \%$ in the band absorbances.
Table 2. Peak positions, vibration modes, and molecule assignments of newly formed IR bands after irradiation of formamide at $20 \mathrm{~K}$ with $200 \mathrm{keV} \mathrm{H}^{+}$ions.

\begin{tabular}{lll}
\hline \hline $\begin{array}{c}\text { Peak position } \\
\mathrm{cm}^{-1} \mu \mathrm{m}\end{array}$ & Vibration & Assignment \\
\hline 33762.96 & $\mathrm{~N}-\mathrm{H}$ stretch & $\mathrm{NH}_{3}$ \\
32063.12 & $\mathrm{~N}-\mathrm{H}$ stretch & $\mathrm{NH}_{4}^{+}$ \\
30743.25 & $\mathrm{~N}-\mathrm{H}$ stretch & $\mathrm{NH}_{4}^{+}$ \\
23424.27 & $\mathrm{C}=\mathrm{O}$ stretch & $\mathrm{CO}_{2}$ \\
22604.42 & $\mathrm{~N}=\mathrm{C}=\mathrm{O}_{\text {ssym }}$ stretch & $\mathrm{HNCO}$ \\
22384.47 & $\mathrm{~N}=\mathrm{N}$ stretch & $\mathrm{N}_{2} \mathrm{O}$ \\
21654.62 & $\mathrm{~N}=\mathrm{C}=\mathrm{O}_{\text {asym }}$ stretch & $\mathrm{OCN}^{-}$ \\
21404.67 & $\mathrm{C} \equiv \mathrm{O}$ stretch & $\mathrm{CO}$ \\
20834.80 & $\mathrm{C} \equiv \mathrm{N}$ stretch & $\mathrm{CN}^{-}$ \\
19975.01 & $\mathrm{Si}-\mathrm{H}$ stretch & $\mathrm{SiH}^{+}$ \\
14786.77 & $\mathrm{~N}-\mathrm{H}_{\text {sym bending }}$ & $\mathrm{NH}_{4}^{+}$ \\
\hline
\end{tabular}

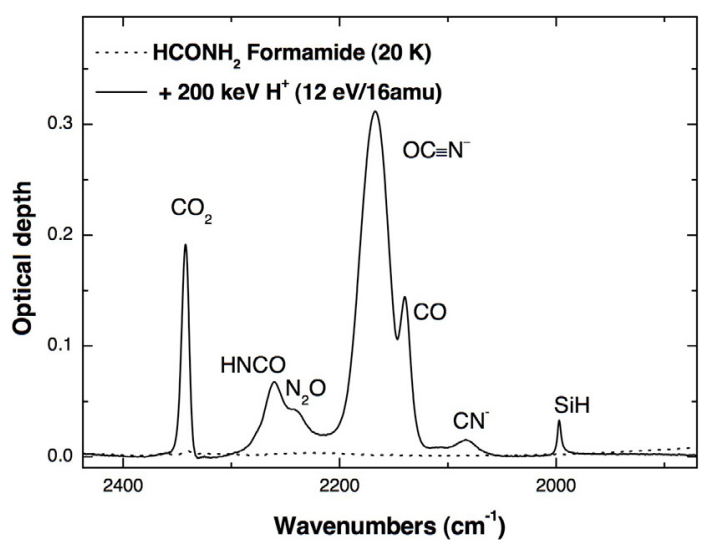

Fig. 2. IR transmittance spectrum in optical depth scale of formamide $(20 \mathrm{~K})$ after irradiation at a dose of $12 \mathrm{eV} / 16$ amu with $200 \mathrm{keV}$ $\mathrm{H}^{+}$ions (full line). The spectrum obtained before irradiation is also shown (dotted line).

\subsection{Ion-induced synthesis of molecules}

Figure 1 also shows the IR spectrum of formamide after $200 \mathrm{keV}$ proton irradiation at $12 \mathrm{eV} / 16 \mathrm{amu}$ dose. The peak positions and identification of each new molecular species is reported in Table 2.

In the 2430-1850 $\mathrm{cm}^{-1}$ spectral region shown better in Fig. 2, both $\mathrm{CO}$ and $\mathrm{CO}_{2}$ absorption bands are observed at 2140 and $2342 \mathrm{~cm}^{-1}$, respectively. The band observed at $2260 \mathrm{~cm}^{-1}$ is assigned to the NCO stretching mode of isocyanic acid (HNCO). The band peaked at wavenumbers larger than that observed by Raunier et al. (2004) $\left(2252 \mathrm{~cm}^{-1}\right)$. This may be due to the presence of a shoulder at $2238 \mathrm{~cm}^{-1}$ attributed to the synthesis of $\mathrm{NO}_{2}$. The weak band at $2083 \mathrm{~cm}^{-1}$ is due to the cyanate anion $\mathrm{CN}^{-}$stretching mode. A shoulder at about $2100 \mathrm{~cm}^{-1}$ is present and tentatively assigned to hydrogen cyanide ( $\mathrm{HCN})$. The presence of the $\mathrm{SiH}$ stretching band observed at $1997 \mathrm{~cm}^{-1}$ stems from the fraction of protons that entirely cross the ice sample and is implanted into the silicon substrate.

Another intense band located at $2165 \mathrm{~cm}^{-1}$ is commonly assigned to cyanate anion $\mathrm{OCN}^{-}$(Grim \& Greenberg 1987; Hudson et al. 2001; Broekhuizen et al. 2004), even if other carriers have been proposed by Pendleton et al. (1999). Evidence that the ammonium cyanate complex $\mathrm{NH}_{4}^{+} \mathrm{OCN}^{-}$is formed is given by the wide band at $1478 \mathrm{~cm}^{-1}$, which is ascribed to $\mathrm{NH}_{4}^{+}$(Raunier et al. 2003). Further evidence of ammonium ion synthesis is formed in the smooth and weak peaks at 3074 


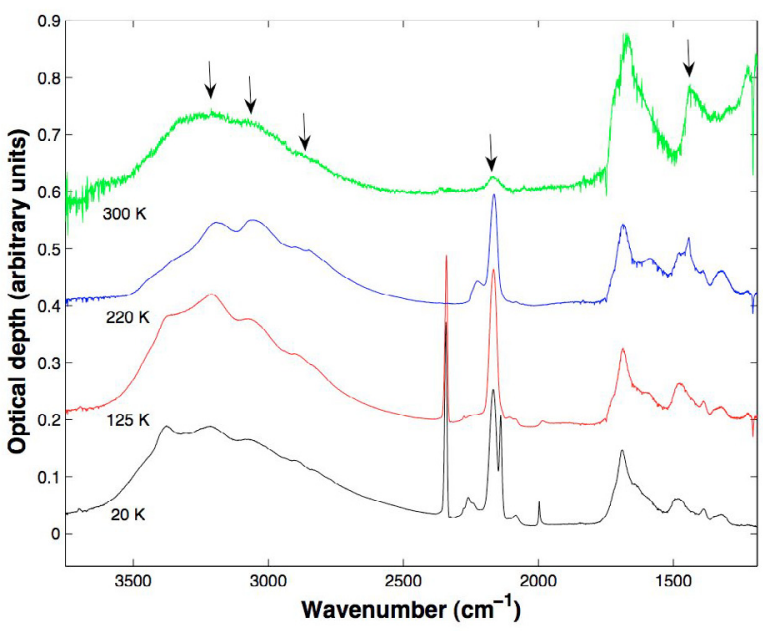

Fig. 3. Comparison of the spectra of (from the bottom to the top): formamide after irradiation with a dose of $24 \mathrm{eV} / 16$ amu at $20 \mathrm{~K}$, and after warming up at $125 \mathrm{~K}, 220 \mathrm{~K}$ and room temperature (this spectrum was magnified 5 times). The arrows show the bands corresponding to the presence of $\mathrm{NH}_{4}^{+} \mathrm{OCN}^{-}$. Spectra have been arbitrarily shifted for sake of clarity.

and $3206 \mathrm{~cm}^{-1}$ observed over the very broad feature extending from about $3600 \mathrm{~cm}^{-1}$ to $2400 \mathrm{~cm}^{-1}$. This feature is due to the overlapping of a number of $\mathrm{NH}$ and $\mathrm{CH}$ stretches of newly formed compounds and of a residual amount of formamide still present after ion irradiation. A tentative identification of $\mathrm{NH}_{3}$ molecules is made by the band present at $3376 \mathrm{~cm}^{-1}$ due to NH vibration stretch and by a noisy feature at about $1110 \mathrm{~cm}^{-1}$ that is not reported in Fig. 1.

\subsection{Temperature effects}

After irradiation, the samples were warmed up (about $1 \mathrm{~K} / \mathrm{min}$ ) to study the evolution of the IR spectrum with temperature, a circumstance that induces a differential sublimation of volatiles and an increase in the synthesized yields likely to be affected by higher mobility. In Fig. 3 the IR spectra in the range $3700-1200 \mathrm{~cm}^{-1}$ of formamide after irradiation at a dose of $24 \mathrm{eV} / 16 \mathrm{amu}$ at $20 \mathrm{~K}$ and after warming up at $125 \mathrm{~K}, 220 \mathrm{~K}$, and $300 \mathrm{~K}$ are shown. Spectra were arbitrarily shifted and, moreover, that at room temperature magnified five times for sake of clarity.

The peak position of $\mathrm{OCN}^{-}$shifts during warm-up from $2167 \mathrm{~cm}^{-1}$ at $20 \mathrm{~K}$ to $2164 \mathrm{~cm}^{-1}$ at $220 \mathrm{~K}$. At room temperature, the $\mathrm{OCN}^{-}$peak position is about $2167 \mathrm{~cm}^{-1}$. The peak position dependence on temperature of $\mathrm{OCN}^{-}$synthesized by ion irradiation of $\mathrm{C}, \mathrm{N}$, O-bearing ice mixtures is a commonly observed phenomena. Nevertheless the amount of shift and the trend observed with temperature depend strongly on the specific ice mixture (Palumbo et al. 2000; Palumbo et al. 2004). In particular the peak position of the $\mathrm{OCN}^{-}$feature observed in the organic residues at room temperature can vary from 2150 to $2168 \mathrm{~cm}^{-1}$ depending on the particular ion-irradiated ice mixture considered (Palumbo et al. 2004). A shift at lower wave number is observed for the $\mathrm{N}-\mathrm{H}_{\text {sym }}$ bending band peaking at $1437 \mathrm{~cm}^{-1}$ at $300 \mathrm{~K}$.

It is evident that the volatile species are desorbed and the bands of ammonium cyanate are left over. The formation of $\mathrm{NH}_{4}^{+} \mathrm{OCN}^{-}$agrees with that measured by the reaction at $10 \mathrm{~K}$ of co-deposited $\mathrm{NH}_{3}$ and HNCO (Raunier et al. 2004). The band strength of ammonium cyanate increases with temperature, which could be due to additional formation by reaction between $\mathrm{NH}_{3}$ and $\mathrm{HNCO}$ synthesized at low $T$. Further features observed in Fig. 3 at $220 \mathrm{~K}$ correspond to crystalline formamide still present in the sample after irradiation, which has had a structural transition at about $180 \mathrm{~K}$.

\section{Discussion}

Formamide has been tentatively identified in the ISO-SWS infrared spectra of different astronomical environments. Although it has been synthesized by UV photolysis and proton irradiation of HCN containing ices (Gerakines et al. 2004) or by UV irradiation of $\mathrm{HNCO}$ ice (Raunier et al. 2004) and $\mathrm{H}_{2} \mathrm{O}, \mathrm{CO}, \mathrm{NH}_{3}$ ice mixtures (Demyk et al. 1998; Broekhuizen et al. 2004), it has not been possible to precisely determine the amount of synthesized (or observed) formamide because of the lack of measured integrated absorbances. This work covers such a need by measuring the integrated absorbances for all the principal features of frozen amorphous pure formamide deposited at $20 \mathrm{~K}$.

With the hypothesis that solid formamide is present in protostellar sources as might be the case for NGC 7538 IRS9 (Raunier et al. 2004), the experimental results reported here on the ion processing of formamide ice have to be considered when astronomical and laboratory spectra are compared. In fact, the evolution of solids (ices, silicates, and carbonaceous phases) in the circumstellar and interstellar media is governed by a number of processes, such as surface reactions, UV photolysis, particle irradiation, and thermal annealing.

Irradiation in the circumstellar environments of young stars or in the dense interstellar medium was simulated by irradiating pure formamide layers at $20 \mathrm{~K}$. After irradiation, warming the target up causes the sublimation of volatile molecules. This simulates the thermal processing suffered by the dust near a forming star or the passage of the dust from a dense to a diffuse medium. It is important to note that in this case, calculations indicate that in the diffuse interstellar medium the temperature of the dust is still of the order of $10 \mathrm{~K}$, as it is in the dense medium (Greenberg 1971; Mathis et al. 1983); however, the gas density is such that icy mantles cannot be maintained or formed.

The flux of low-energy cosmic rays irradiating grains in the interstellar medium is not well known, although a reasonable estimate was given by Moore (1999). She calculated that in cold dense clouds, ions deposit about $30 \mathrm{eV} /$ molecule (in $10^{8}$ years), a factor of 10 less than in the diffuse medium. The dominant contribution of cosmic rays comes mainly from low-energy protons in the $\mathrm{MeV}$ range, which lose energy through ionizations and excitations of the target atoms as $200 \mathrm{keV} \mathrm{H}^{+}$ions do. From the present experimental results we estimated that 64 and $78 \%$ of the formamide molecules are destroyed at 12 and $24 \mathrm{eV} / 16 \mathrm{amu}$, respectively. This result indicates that the destruction of formamide is a first-order process. Extrapolating to the irradiation dose expected in the dense medium, it is possible to infer that, in first approximation, about $20 \%$ of the frozen formamide molecules are able to survive in dense medium on a time scale of $10^{8}$ years.

A very important result is that the species produced after irradiation of formamide are mostly the same as those produced in a large number of irradiation experiments conducted by different groups on different icy mixtures containing simple $\mathrm{H}, \mathrm{O}$, $\mathrm{C}$, and $\mathrm{N}$ bearing molecules (Grim et al. 1987; Demyk et al. 1998; Moore \& Hudson 2003; Hudson et al. 2001; Palumbo et al. 2000, 2004). Moreover, most of the bands shown in Fig. 2 have been observed in astronomical spectra (Sandford et al. 1990; 
Elsila et al. 1997; Novozamsky et al. 2001; Pendleton et al. 1999; Tegler et al. 1993, 1995; van Broekhiuzen 2005). Of particular interest is the synthesis of the ammonium cyanate molecule. In fact the $\mathrm{CN}$ stretching in $\mathrm{OCN}^{-}$is considered responsible for the $2165 \mathrm{~cm}^{-1}$ band observed in almost all the observations of molecular clouds and in young stellar objects. Ion and UV processing of ice samples containing $\mathrm{N}$-bearing molecules such as $\mathrm{N}_{2}, \mathrm{NH}_{3}, \mathrm{HCN}$, and $\mathrm{HNCO}$ mixed with the most abundant molecules observed in space $-\mathrm{H}_{2} \mathrm{O}, \mathrm{CO}, \mathrm{CO}_{2}$, and $\mathrm{CH}_{3} \mathrm{OH}-$ share the yielding of $\mathrm{NH}_{4} \mathrm{OCN}^{-}$with the results of this work.

Ammonium cyanate is theoretically very important since the first synthetic production of an organic from inorganic compounds (Wohler 1828). Once prepared by the gaseous reaction of ammonia and cyanic acid, thermal annealing of ammonium cyanate aqueous solution formed urea $\left(\mathrm{NH}_{2}\right)_{2} \mathrm{CO}$ through dissociation into ammonia and isocyanic acid (Warner \& Stitt 1933). Urea was also synthesized at $10 \mathrm{~K}$ by UV irradiation of isocyanic acid and tentatively detected in a protostellar object (Raunier et al. 2004).

These results suggest that the chemistry of HCN (in the presence of $\mathrm{H}_{2} \mathrm{O}$ ) has to be considered a preferential route for the prebiotic chemistry even in space.

The plausible pathways for the synthesis of biomolecules in space at low temperature have to be deal with when studying the organic compounds that might have favoured the emergence of life when delivered on the early Earth by comets, meteorites and IDPs. Ion and photon processing of ices containing $\mathrm{H}, \mathrm{C}, \mathrm{N}$, and $\mathrm{O}$ shows a specificity in driving the chemistry in space so that it requires deep investigation, in particular for its important consequences on astrobiological research.

Acknowledgements. This research has been supported by the Italian Space Agency (ASI), and the Italian Ministero dell'Istruzione, Università e Ricerca (MIUR).

\section{References}

Baratta, G. A., \& Palumbo, M. E. 1998, J. Opt. Soc. Am. A, 15, 3076 Bernstein, M. P., Sandford, S. A., Allamandola, L. J., Chang, S., \& Scharberg, M. A. 1995, ApJ 454, 327

Bockelée-Morvan, D., Lis, D. C., Wink, J. E., et al. 2000, A\&A, 353, 1101

van Broekhuizen, F. A., Keane, J. V., \& Schutte, W. A. 2004, A\&A, 415, 425

van Broekhiuzen, F. A., Pontoppidan, K. M., Fraser, H. J., \& van Dishoeck, E. F. 2005, A\&A, 441, 249

Cases, A. M., Gomez Marigliano, A. C., Bonatti, C. M., \& Solimo, H. N. 2001, J. Chem. Eng. Data, 46, 712

Chyba, C. F., \& Sagan, C. 1992, Nature, 255, 125
Chyba, C. F., \& McDonald, G. D. 1995, Annu. Rev. Earth Planet. Sci., 23, 215 Demyk, K., Dartois, E., d'Hendecourt, L., et al. 1998, A\&A, 339, 553 Elsila, J., Allamandola, L. J., \& Sandford, S. A. 1997, ApJ, 479, 818 Eschenmoser, A., \& Loewenthal, E. 1992, Chem. Soc. Rev., 1, 16 Gerakines, P. A., Moore, M. H., \& Hudson, R. L. 2004, Icarus, 170, 202 Greenberg, J., \& Mayo 1971, A\&A, 12, 240

Grim, R. J. A., \& Greenberg, J. M. 1987, ApJ, 321, L91

Hudson, R. L., Moore, M. H., \& Gerakines, P. A. 2001, 550, 1140

Mathis, J. S., Mezger, P. G., \& Panagia, N. 1983, A\&A, 128, 212

McNaughton, D., Evans, C. J., Lane, S., \& Nielsen, C. J. 1999, J. Mol. Spectr., 193,104

Millar, T. J. 2004, Organic molecules in the interstellar medium, in Astrobiology: future perspectives, ed. P. Ehrenfreund, W. Irvine, T. Owen, L. Becker, J. Blank, J. R. Brucato, L. Colangeli, S. Derenne, A. Dutrey, D. Despois, A. Lazcano, \& F. Robert (Kluwer Academic Publisher), 305, 17

Miller, S. L. 1953, Science, 117, 528

Moore, M. H. 1999, The Physics and Chemistry of Ice in the Interstellar Medium, in Solid Interstellar Matter: the ISO Revolution, ed. L. d'Hendecourt, C. Joblin, \& A. Jones (Berlin: Springer-Verlag), 199

Moore, M. H., \& Hudson, R. L. 2003, Icarus, 161, 486

Novozamsky, J. H., Schutte, W. A., \& Keane, J. V. 2001, A\&A, 379, 588

Oparin, A. I. 1938, The Origin of Life, New York (McMillan Publishing)

Palumbo, M. E., Strazzulla, G., Pendleton, Y. P., \& Tielens, A. G. G. M. 2000, ApJ, 534, 801

Palumbo, M. E., Ferini, G., \& Baratta, A. 2004, A\&A, 33, 49

Pendleton, Y. J., Tielens, A. G. G. M., Tokunaga, A. T., \& Bernstein, P. A. 1999, ApJ, 513, 294

Raunier, S., Chiavassa, T., Duvernay, F., et al. 2004, A\&A, 416, 165

Rubalcava, H. 1956, Ph.D. Thesis, Caql. Inst. Of Tech. Pasadena, California, http://resolver. caltech. edu/CaltechETD: etd-06242004-155629

Saladino, R., Ciambecchini, U., Crestini, C., et al. 2003, ChemBiochem, 4, 514

Saladino, R., Crestini, C., Costanzo, G., \& Di Mauro, E. 2004, Cour. Org. Chem., 8,1425

Saladino, R., Crestini, C., Neri, V., et al. 2005, ChemBioChem, 6, 1368

Sandford, S. A., \& Allamandola, L. J. 1990, ApJ, 355, 357

Schutte, W. A., Boogert, A. C. A., Tielens, A. G. G. M., et al. 1999, A\&A, 343, 966

Strazzulla, G., Baratta, G. A., \& Palumbo, M. E. 2001, Spectrochim. Acta, A, 57,825

Tegler, S. C., Weintraub, D. A., Allamandola, L. J., et al. 1993, ApJ, 411, 260

Tegler, S. C., Weintraub, D. A., Rettig, T. W., et al. 1995, ApJ, 439, 279

Warner, J. C., \& Stitt, F. B. 1933, J. Chem. Soc., 55, 4807

Westley, M. S., Baratta, G. A., \& Baragiola, R. A. 1998, J. Chem. Phys., 108, 3321

Willner, S. P., Gillett, F. C., Herter, T. L., et al. 1982, ApJ, 253, 174

Wohler, F. 1828, Berzelius Jahresberichte, 12, 266

Wood, B. E., \& Roux, J. A. 1982, J. Opt. Soc. Am., 72, 720

Yamada, H., \& Okamoto, T. 1972, Chem. Pharm. Bull., 20, 623

Yamada, H., Okamoto, Y., \& Yakugaku, Z. 1975, 95, 493

Ziegler, J. F. 1977, The Stopping and Range of Ions in Matter. V. 2-6 (New York: Pergamon Press)

Ziegler, J. F., Biersack, J. P., \& Littmark, U. 1996, The Stopping and Range of Ions in Solids (New York: Pergamon Press) 